Instituto Internacional de Investigación y Desarrollo Tecnológico Educativo INDTEC, C.A.

DOI: https://doi.org/10.29394/Scientific.issn.2542-2987.2020.5.18.10.193-212

OAI-PMH: http://www.indteca.com/ojs/index.php/Revista Scientific/oai

Artículo Original / Original Article

\title{
Diagnóstico de factores de riesgo de niños en albergue: Una propuesta estratégica para su desarrollo integral
}

\author{
Autora: Mariela González López \\ Universidad Pedagógica Nacional del Estado de Chihuahua, UPNECH \\ mgonzalez.ijd@upnech.edu.mx \\ Chihuahua, México \\ https://orcid.org/0000-0002-3178-8000
}

\section{Resumen}

Es una propuesta de tipo cualitativa mediante un diagnóstico preventivo en albergue situado en Chihuahua, cuyo objetivo es realizar un currículo para atender y garantizar los derechos de los niños y niñas de 0 a 6 años, hijos de los trabajadores que se hospedan en el lugar, en cuanto a su desarrollo integral, como una oportunidad de prevención y cumplimiento de sus derechos. Esta investigación se llevó a cabo en tres etapas: 1. diseño de diagnóstico de factores de riesgo de salud, cognitivo y educativo para albergue; 2. Aplicación de diagnóstico en albergue; y 3. Conclusiones, las personas que se alojan, en su mayoría son indígenas que utilizan su lengua materna, con raíces bien arraigadas, en la cual los lleva a estos albergues por tener un trabajo digno para la sobrevivencia familiar, y por ende los niños son vulnerables en esas circunstancias para su desarrollo integral, por lo tanto, las estrategias son de cuidados integrales para la niñez.

Palabras clave: infancia; derechos humanos; enseñanza.

Cómo citar este artículo:

González, M. (2020). Diagnóstico de factores de riesgo de niños en albergue: Una propuesta estratégica para su desarrollo integral. Revista Scientific, 5(18), 193-212, e-ISSN: 2542-2987. Recuperado de: https://doi.org/10.29394/Scientific.issn.2542-2987.2020.5.18.10.193-212

Fecha de Recepción: 06-06-2020
Fecha de Aceptación: 17-09-2020
Fecha de Publicación: 05-11-2020 
OAI-PMH: http://www.indteca.com/ojs/index.php/Revista_Scientific/oai

Artículo Original / Original Article

Diagnosis of risk factors of children in shelters: A strategic proposal for their integral development

\begin{abstract}
It is a qualitative proposal through a preventive diagnosis in a shelter located in Chihuahua, whose objective is to carry out a curriculum to attend and guarantee the rights of children from 0 to 6 years old, children of workers who stay in the place, as for its integral development, as an opportunity for prevention and fulfillment of their rights. This research was carried out in three stages: 1 . design of diagnosis of health, cognitive and educational risk factors for shelter; 2. Application of diagnosis in shelter; and 3. Conclusions, the people who stay, are mostly indigenous people who use their mother tongue, with wellrooted roots, in which it takes them to these shelters for having a decent job for family survival, and therefore the children They are vulnerable in these circumstances for their integral development, therefore, the strategies are of integral care for children.
\end{abstract}

Keywords: childhood; human rights; teaching.

How to cite this article:

González, M. (2020). Diagnosis of risk factors of children in shelters: A strategic proposal for their integral development. Revista Scientific, 5(18), 193-212, e-ISSN: 2542-2987. Recovered from: https://doi.org/10.29394/Scientific.issn.2542-2987.2020.5.18.10.193-212

Date Received: 06-06-2020
Date Acceptance:

17-09-2020
Date Publication:

05-11-2020 


\section{Introducción}

En la actualidad el tema infantil, sigue siendo un asunto visible y que hay que apostarle para que reciban un desarrollo integral para sus vidas del presente para un futuro prometedor. Es decir, si los atendemos con verdadera atención de formación académica, física, mental, psicológica y social, mediante técnicas de juego y estrategias que hagan crecer cognoscitivamente tendremos niños, niñas y adolescentes con una infancia exitosa. Además, para el desarrollo familiar, económico, social y cultural del país. Cada año por largos periodos de tiempo, hombres, mujeres y familias enteras viajan por trabajo a los campos del Estado de Chihuahua, por un trabajo digno para vivir.

Los trabajadores viajan con hijos menores de edad, de las cuales no reciben una educación integral por el trabajo de los padres de 8 a 12 horas diarias de su tiempo laboral. Dichas instancias donde llegan los trabajadores de otros municipios del país hasta de otros países de lengua hispana y lengua indígena, por un lado, es vital y necesario que se apliquen medidas de seguridad del menor, alimenticia, escolar y de higiene. Por el otro es importante hacer un enfoque analítico crítico en la asistencia integral de todos ellos, que llegan a los albergues como garantía de sus derechos.

La educación juega un papel importante en su desarrollo, los programas fuera de la escuela son importantes para proporcionar oportunidades de aprendizaje adaptables, nuevas habilidades y conocimientos a un gran porcentaje de personas que están fuera del alcance de la educación formal. Por su parte, Chacón-Ortiz (2015): señala que, la educación no escolarizada es una formación para que crezca el estudiantado, nace como una alternativa para solucionar problemas, por consecuente la educación escolarizada no ha cumplido. La educación inicial no formal (EI), lo conforman los programas de formación de competencias o habilidades productivas y constructivas, que se dan a los tutores de los niños y niñas de cero a seis años de edad, para su fortalecimiento en el crecimiento tanto parentales como la integral del niño. 
Se establece una estructura para su enseñanza y una metodología para ponerla en marcha, a los tutores de los niños y niñas. Para definir el currículo que se diseñó en esta investigación, Iriondo y Gallego (2013): refieren a los factores socioeconómicos, políticos y culturales del currículo comprendido en los contenidos que se deben enseñar o aprender, una planeación pedagógica para el desarrollo integral de los niños y niñas, que deben ser vividas por los niños albergados.

El albergue es un lugar para proteger a las personas, comúnmente son lugares en la que sí pueden pagar las personas con bajos recursos en la estancia de un trabajo fuera de su lugar natal. En Chihuahua, existen varios albergues, en la que se brinda un espacio económico para que las personas descansen y estén seguras durante el tiempo de cosecha.

La situación aquí es que se quedan muchos niños en el asilo, y muchas de las veces no hay cómo atender a tantos niños, porque no hay doctores, maestros o personal especializado todo el tiempo de estancia de esos trabajadores, por consecuente este currículo atenderá necesidades que se deben cubrir a los niños y niñas que se quedan en el albergue y sean atendidos como un derecho.

En este estudio académico, se diseñó un currículo de educación inicial para el desarrollo integral de los menores en diferentes componentes. Lo anterior puede ser implementado para todo tipo de necesidades que haya en las sociedades comúnmente de bajos recursos, por ejemplo, los pueblos indígenas, las zonas rural-urbanas para centros de guarderías, para centros comunitarios que hay en todos los municipios de los estados mexicanos, además de los albergues. Todo lo anterior es para su correcta nutrición, desarrollo social, cognitivo y de salud.

El concepto de Educación inicial es reciente, una secuela de la mejora en los estilos de vida de las sociedades occidentales, más desarrolladas, cuyos pobladores se han visto la tarea de estructurar sitios continuos de 
estudios y capacitación a lo largo de la vida.

Según la Ley General de los Derechos de Niñas, Niños y Adolescentes (2018): los niños tienen derecho a la vida, a la supervivencia y al desarrollo, tal como se describe en el capítulo primero del artículo 14-16. Por su parte el Fondo de las Naciones Unidas para la Infancia (UNICEF, 2018a): se enfoca en que los niños y las niñas deben tener una vida con cuidados tanto físicas y cognitivas para un desarrollo integral.

Además, apostar por los niños es una vía para la recuperación y desarrollo integral de los infantes, por consiguiente, este currículo se fundamenta a lo que llama I el Fondo de las Naciones Unidas para la Infancia (UNICEF, 2018b): la protección de los niños y niñas es prioridad para todo.

Los primeros momentos de los niños y niñas sí importan en la manera que garanticen a los niños un comienzo favorable para su vida $y$, ampliar servicios fundamentales en la niñez, en donde vive, y los centros de salud, colegios y agrupaciones en la que se brinde calidad para su desarrollo. Además, liderar la orientación a programas de desarrollo de los niños y niñas más necesitados. Se enfocó en fomentar la lactancia materna y el desarrollo infantil temprano. Para estola Universidad cumple con la formación de estudiantado para su preparación en la educación de la primera infancia.

La Asociación Mundial de Educadores Infantiles (2006a): menciona la educación de la niñez, que determinala línea escolarizada, sino también por la vía no convencional. De la cual se puede implementar la educación formal y no formal en los albergues, casa de cuidado, casas hogar, cárceles y otros, por consiguiente, muchas de las veces los niños y niñas de cero a seis años no tienen educación formal dentro de los albergues en Chihuahua, de la cual se propone un currículo para la intervención en educación inicial por la vía no formal.

En este estudio se diseñaron las características que debe llevar un programa de para llevarlo a cabo. Además, se propone como implementarlo 
en albergues donde se encuentran migrantes con sus hijos que no reciben una educación formal. Es una propuesta diagnóstica de un albergue situado en Chihuahua, para los hijos de los trabajadores en su mayoría indígenas que trabajan en los campos chihuahuenses en México.

El interés específico es que se brinde la seguridad a los niños y niñas que llegan a esos campos. Dicho trabajo se realizó como parte de las estrategias, que se aplican en la materia de educación inicial por la vía no formal que se imparte por medio de la Universidad Pedagógica Nacional de Chihuahua.

En el albergue no hay docentes a la falta de inversión del mismo, por consecuente es fundamental para una educación integral y derechos de la infancia. Después de los resultados del diagnóstico, se realizó un currículo por la vía no formal para que sean atendidos, por todo el tiempo en que los padres trabajan en los campos chihuahuenses. Por consiguiente, es una propuesta para fortalecer los lazos parentales, desarrollo físico, psicológico, alimenticio y cognitivo de los niños y niñas.

\subsection{Fundamentación del programa de educación inicial no formal}

La educación no formal, para Cabalé y Rodríguez (2017): es una forma de contribuir socialmente y educativamente para el desarrollo integral de las personas. La educación no formal es para el desarrollo sostenible, tiene un valor social para las comunidades, apoya a los que menos tienen, como las personas étnicas, agrega valor económico al país, sobre todo da un beneficio a los que toman los programas por esta vía no formal, para el desarrollo de los niños y niñas, además para dar cumplimiento de los derechos de los infantes.

La educación no formal está relacionada a lo que menciona el Banco Mundial (2018): en el año 2015 disminuyó la pobreza a un 8\%, sin embargo, tenían considerado que en el año 2030 se acabaría, hay en la actualidad, casi 1100 millones de individuos son de bajos recursos, en comparación a finales 
del siglo XIX. Hace cinco años había millones de ellos que sobrevivían con menos de 40 pesos al día. Además, para que podamos terminar con la pobreza tenemos que encontrar ideas en el cual ayude a las personas a salir de la pobreza o ayudar en el proceso de las personas en recuperación de su estatus económico, social, cognitivo, alimenticio y de salud.

Cabe destacar que para la Asociación Mundial de Educadores Infantiles (2006b): la educación inicial no formal genera progreso en vitalidad, nutrición, hábitos, previsión familiar y sociedad, que presentan dictámenes favorables, procedentes de la participación de los proyectos. Los programas son inmediatos para salvar vidas, salvar a niños del fracaso escolar, además que los programas son para padres e hijos. Por lo tanto, Eming y Fujimoto (2004): realizan proyectos de educación no formal en Educación Inicial, la cual simbolizan alternativas para desarrollar la habilidad de las naciones en la contienda contra la pobreza.

Una evidencia de que debemos implementar currículos no formales para las personas que está en situación de pobreza, salen de sus pueblos natales para trabajar en otros Estados de la República por periodos largos, para ganarse la vida y poder alimentar a sus familias. Además, con bajos sueldos que apenas alcanza para comer. Sin embargo, en donde entra el trabajo profesional de la investigadora o maestras, es cuando proponen un currículo para todos los niños y niñas que ingresan en un albergue, para que lo lleven a cabo los cuidadores o los pedagogos encargados.

\section{Metodología (Materiales y métodos)}

Es el diseño y aplicación de un diagnóstico educativo en un albergue y después se realiza una propuesta para dar seguridad a los niños y niñas de cero a 6 años. Mencionado a Capurro y Rada (2007): el diagnóstico es una propuesta para el albergue situado en Chihuahua, la cual consiste en dar estrategias para coadyuvar en el cuidado integral de los niños en el albergue, 
apoyando en la parte de derechos humanos; enseñanza y alimentación. Como se expresa en la figura 1, el procedimiento de la metodología es la siguiente: Figura 1. Metodología para la elaboración de la propuesta para albergue por la vía no formal.

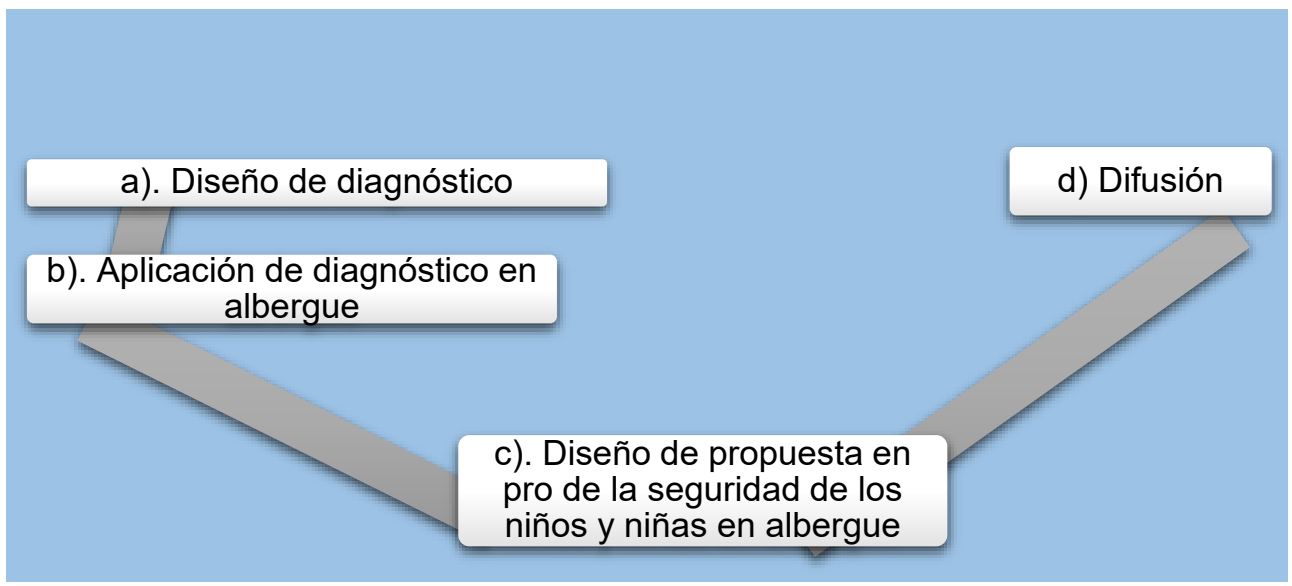

Fuente: La Autora (2020).

Para realizar un currículo por la vía no formal para niños de cero a seis años de edad, los resultados del diagnóstico en el Albergue fue el siguiente: hay jornaleros migrantes, ubicado en Chihuahua, este albergue refugia a migrantes originarios principalmente de la sierra tarahumara del Estado y en ocasiones también alberga a migrantes de otros Estados como Sinaloa.

Estos migrantes vienen a establecerse para trabajar principalmente en el área agrícola, por temporadas largas, teniendo un establecimiento aproximadamente de seis a ocho meses. Los refugiados pagan una cuota de $\$ 110.00$ pesos semanales para el sustento y cuidado de la entidad. El albergue cuenta con poco apoyo del gobierno y se encuentra en situaciones un poco desfavorables en infraestructura y existe mucha falta de información a padres de familia sobre la importancia del desarrollo integral de sus hijos.

El albergue cuenta con cuarenta y cinco (45) cuartos individuales, existe una "guardería" en donde asisten cinco infantes con edades de 4 meses a 4 
años, y un salón para impartir clases, donde asisten de 5 a 10 niños con edades de 6 a 11 años. Cuenta además con un módulo familiar, sanitarios, un comedor que actualmente no está en función, cancha de futbol rápido, cerco perimetral y área de juegos. La encargada de los niños más pequeños tiene una capacitación para el cuidado de los niños, además de tener un certificado en Enfermería Auxiliar. El profesor encargado de impartir las clases tiene una Licenciatura en Educación Indígena.

\section{Resultados}

A continuación, se detalla la propuesta, la cual se comparte a los encargados del albergue. La propuesta de un currículo de educación inicial por la vía no formal sobre el cuidado de la alimentación de niños de cero a seis años de edad. En la que se determina una necesidad vital para su desarrollo integral bajo el cuidado de personas en un albergue, bajo los cuidados en la que brindará la garantía de derechos.

Este programa consiste en informar a los padres de familia para ayudar a que los infantes tengan una mejor alimentación y una vida a largo plazo saludable, asegurando uno de sus derechos de la infancia. Lo primero será hablarles en qué consisten los hábitos alimenticios ya que estén informados del tema, a los padres de familia se les recomendará realizar la pirámide del buen comer para que sepan cómo están clasificados los alimentos y qué importancia tiene conocerla, para así saber llevar una dieta saludable y balanceada además de que sepan cómo propiciar hábitos saludables en la primera infancia.

Así mismo se les pondrá a realizar platillos con un precio accesible para que se den cuenta que comer bien no es sinónimo de alto costo. Con el objetivo que las personas de escasos recursos tengan un conocimiento más amplio de la importancia de la alimentación, basándonos en la primera infancia con niños de 0-6 años de edad. Además de que conozcan los hábitos 
alimenticios y alimentos determinantes durante su crecimiento. El currículo siguiente es para fortalecer el desarrollo integral de los niños en el albergue, la cual se proponen los siguientes componentes La alimentación, aprendizaje de las matemáticas por medio del juego, actividades para el desarrollo psicomotor, la lectura, la lectoescritura y cuidados de higiene.

\subsection{Alimentación}

La hipótesis de las necesidades humanas que de acuerdo con Maslow (1943): se fundamentan en la motivación humana, la nutrición aparece en la base de la pirámide de necesidades fundamentales, junto con aspirar, reposar, y sostener un balance en nuestro ser.

La lactancia materna es la primera alimentación que tiene un infante, se recomienda por motivos neurológicos, orgánicos y sensoriales. Los expertos Brahm y Valdés (2017a): dicen que no hay problema en que se continúe el amamantamiento hasta los 2 años, ya que es beneficioso para la progenitora y el infante desde la perspectiva de vida del individuo. El amamantamiento puede prever la muerte infantil en el mundo, y disminuir el riesgo de muerte súbita del bebe.

En este sentido, Brahm y Valdés (2017b): exponen que "las fórmulas lácteas y mamaderas tienen riesgos inherentes asociados, hay riesgo de complicaciones de la cavidad oral, tales como respiración bucal, maloclusión, alteración de la mordida y caries, lo anterior se ven afectadas negativamente en el bebé" (pág. 88).

Paralelamente, la familia del infante es la responsable del desarrollo integral de sus hijos, para un crecimiento efectivo en la edad temprana. Lo anterior está relacionada con los hábitos de la alimentación, construcción social y cultural. Por consiguiente, un buen habito alimenticio es en la familia con los padres, es por eso que con este programa se informen acerca de cómo mantener una buena alimentación con los niños por medio de hábitos 
saludables. Empezaremos diciéndoles en que consiste un hábito, en la formación de los hábitos comienza en la vida diaria del niño con sus rutinas, esto hace referencia a las costumbres que se tenga en la familia que pertenece el niño, por ejemplo, en cuestión de la alimentación es que comen a diario los padres, y el niño se basa en que comerá en el albergue.

Los beneficios que trae consigo el tener buenos hábitos alimenticios es principalmente el prevenir enfermedades, logrará que los niños y niñas adquieran mejor aprendizaje, ya que estará bien nutrido, formar rutinas que favorezcan la alimentación y a su vez estarán estimulando actitudes positivas. Un buen hábito alimenticio fomenta el bienestar integral y la seguridad alimentaria de las familias y los niños y niñas que las integran. Ahora bien, basándonos en la alimentación veamos qué es la pirámide alimenticia o bien para que sirve, y con el objetivo de realizar combinaciones de vegetales, cereales y carnes en las tres comidas fuertes.

Tabla 1. Menú para el albergue

\begin{tabular}{|l|l|}
\hline \multicolumn{1}{|c|}{ Dia } & \multicolumn{1}{c|}{ Platillo } \\
\hline Lunes & $\begin{array}{l}\text { Mollete: Pan, jugo de tomate, huevos y queso rallado, junto con un } \\
\text { vaso de agua de mango }\end{array}$ \\
\hline Martes & $\begin{array}{l}\text { Un burrito: Huevos revueltos, frijoles negros y pico de gallo en tortilla } \\
\text { de trigo integral acompañado de un vaso de agua fresca de lima. }\end{array}$ \\
\hline Miércoles & $\begin{array}{l}\text { Huevos cocidos con queso en pan blanco, manzana, y con una taza } \\
\text { de agua de fruta. }\end{array}$ \\
\hline Jueves & $\begin{array}{l}\text { Pancake (Hotcakes) con miel, fresas y un vaso de leche con un vaso } \\
\text { acompañado de agua fresca de naranja natural. }\end{array}$ \\
\hline Viernes & $\begin{array}{l}\text { Pan tostado, un huevo duro, una taza de leche y un tazón de fresas } \\
\text { picadas con un vaso de agua fresca de guayaba natural. }\end{array}$ \\
\hline Sábado & $\begin{array}{l}\text { Sándwich con tocino de pavo, verduras verdes, queso, vaso de agua } \\
\text { de fresa. }\end{array}$ \\
\hline Domingo & Trocitos de pollito, jitomate, pan y un agua fresca de limón. \\
\hline
\end{tabular}

Fuente: La Autora (2020).

Para adecuar la alimentación de cada niño, es preciso conocer las características fisiológicas y del desarrollo en cada etapa, y tener en cuenta el status socio económico de cada familia, sus hábitos, sus costumbres y así adecuarnos a sus necesidades, además tiene que intervenir el Estado como 
garantía de este derecho de los niños y niñas. En la tabla 1, se propone el menú para los niños en cantidades pequeñas: que los padres conozcan los alimentos que deben comer y la importancia de una lonchera saludable.

\subsection{La enseñanza de matemáticas por medio del juego}

Las actividades para aprender matemáticas básicas a niños de cinco y seis años de edad, proponen los juegos de mesa, como el rompecabezas de números del uno al treinta, sumas y restas con materiales que estén alrededor del niño. Por ejemplo, piedras, hojas de papel de colores o blancas, hojas de árboles, ropa, cubiertos de plástico, etc.

\subsection{Desarrollo psicomotor}

En la tabla 2, se observan diferentes actividades para el desarrollo psicomotor; equilibrio, postura, habilidad espacial, destreza de manos, viso manual, en todas las actividades debe estar presente un adulto. Las actividades rutinarias para los niños y niñas, son periodos cortos de aprendizaje y poner en marcha habilidades físicas, psicológicas y motoras tanto gruesa como fina, en la que se desarrollan en distintas edades y etapas.

Tabla 2. Actividades para el desarrollo psicomotor.

\begin{tabular}{|l|l|}
\hline Actividad & Edades \\
\hline Rodar sobre el la cama. & Seis a ocho meses con ayuda. \\
\hline Rodar en el piso. & De uno a seis. \\
\hline Andar en triciclo. & Tres en adelante. \\
\hline $\begin{array}{l}\text { Meter tapas de refresco en un bote donde } \\
\text { quepa su mano. }\end{array}$ & Dos años en adelante. \\
\hline Armar rompecabezas de seis piezas & Dos. \\
\hline Armar rompecabezas de doce a 24 piezas. & Tres. \\
\hline Armar rompecabezas de cien piezas. & Cinco en adelante. \\
\hline Juego con pelota. & Dos a seis. \\
\hline $\begin{array}{l}\text { Destrezas de las manos, abrir, cerrar los puños, } \\
\text { llenar un círculo con bolitas de papel, colocar un } \\
\text { bote de basura y que el niño logre pegarle al } \\
\text { bote a una distancia corta. }\end{array}$ & Dos en adelante. \\
\hline
\end{tabular}




\begin{tabular}{|l|l|}
\hline $\begin{array}{l}\text { Actividades para desarrollar habilidades viso } \\
\text { manual. }\end{array}$ & De seis meses en adelante. \\
\hline
\end{tabular}

Fuente: La Autora (2020).

Las actividades para el desarrollo psicomotor, para Mendoza (2017); y Alonso (2018): son habilidades de cuerpo y mente para desempeñarse y manifestarse en los niños y las niñas. Cabe destacar que estas habilidades deben enseñarse, para que las manifieste el infante.

\subsection{Actividades para la lectura}

Las recomendaciones en el albergue de Chihuahua se recomienda la lectura para leer a los niños y niñas de 0 a 6 años de edad, una vez de 20 a 30 minutos de cuentos infantiles, ya que desarrollan el apego, la escucha, concentración, seguridad, atención y la memoria desde pequeños. Por su parte, Gutiérrez (2018): puntualiza que hay interés por el aprendizaje de la lectura, así como la habilidad de fomentar la adquisición de esta habilidad lingüística en las edades de cinco y seis años de edad, ha sido objeto de numerosas investigaciones recientemente. Los materiales que se pueden utilizar para la lectura con niños y niñas, es importante usar libros con imágenes grandes, lecturas cortas, con letras medianas y grandes.

\subsection{Actividades para la lectoescritura}

A partir de los tres años de edad, se recomienda realizar actividades de lectoescritura debido a su madurez cognitiva. Por lo tanto, para la enseñanza de la lectoescritura, interpretando a Gil (2019): es fundamental para el aprendizaje de la lectura, la estimulación y el desarrollo de la consciencia fonológica. Además, Sarmiento (2018): concluye que a través de la implementación de estrategias pedagógica apoyadas en las tecnologías de la información y la comunicación, fortalece los procesos de lectoescritura de los 
niños y niñas. En la tabla 3, se presentan las actividades para propiciar la lectoescritura en niños de educación preescolar.

Tabla 3. Estrategias para la lectoescritura y su repetición.

\begin{tabular}{|c|c|c|}
\hline & Estrategia & Repetición \\
\hline Lunes & $\begin{array}{l}\text { Cuentas un cuento y dibujas el paisaje o } \\
\text { personajes primarios y que coloreen los } \\
\text { niños. }\end{array}$ & $\begin{array}{l}\text { Diario por un mes, para que se } \\
\text { haga hábito. }\end{array}$ \\
\hline Martes & $\begin{array}{l}\text { Escribir su nombre en grande, le señalas } \\
\text { que dice en una hoja, que peguen, } \\
\text { coloreen su nombre o tomas su mano y } \\
\text { que comience a mantener un lápiz en su } \\
\text { mano para repasar su nombre con } \\
\text { colores. }\end{array}$ & $\begin{array}{l}\text { Dos veces por semana en un } \\
\text { mes. }\end{array}$ \\
\hline Miércoles & $\begin{array}{l}\text { Rasgar papel de revista, modelas y luego } \\
\text { que repita el niño }\end{array}$ & $\begin{array}{l}\text { Cuatro veces por semana en } \\
\text { un mes }\end{array}$ \\
\hline Jueves & $\begin{array}{l}\text { Que haga bolitas de papel con lo que } \\
\text { rasgó y luego las meta en una botella de } \\
\text { plástico. }\end{array}$ & $\begin{array}{l}\text { tres veces por semana en un } \\
\text { mes. }\end{array}$ \\
\hline Viernes & $\begin{array}{l}\text { Saca las bolitas de papel, dibujas un } \\
\text { círculo en el piso y después se separa a } \\
\text { dos metros de distancia para que las lleve } \\
\text { soplando hasta que las meta al círculo. }\end{array}$ & $\begin{array}{l}\text { Cuatro veces por semana en } \\
\text { un mes. }\end{array}$ \\
\hline Sábado & $\begin{array}{l}\text { Escribes palabras en el pizarrón de } \\
\text { nombres que se le hagan comunes a los } \\
\text { niños, luego realizas esas palabras, las } \\
\text { recortas por letra, y luego que los niños y } \\
\text { niñas las repliquen en el pizarrón. }\end{array}$ & $\begin{array}{l}\text { Diario por un mes, para que se } \\
\text { haga hábito. }\end{array}$ \\
\hline Domingo & $\begin{array}{l}\text { Juego con piedras en el patio, en una hoja } \\
\text { escribes su nombre para que el niño } \\
\text { coloque en el contorno de cada letra. }\end{array}$ & Dos veces por semana. \\
\hline
\end{tabular}

Fuente: La Autora (2020).

\subsection{Cuidados de higiene}

Según Faria, Oliveira y Ferreira (2015): puntualizan que la higiene abarca diferentes áreas como higiene colectiva, mental, profesional, social, corporal y alimenticia. En este esta sección refiere a la cuestión corporal y alimenticia. Por su parte, Soto, Gómez, Parrado, Hernández-Rodríguez y Gomez (2017): establecen que, hay una necesidad de monitorear e intervención en hábitos de higiene y cuidado en ambientes para el cuidado de los niños. Es importante recordar que el estado de salud de cada persona 
depende en gran medida de cómo sean sus hábitos alimenticios.

Un hábito es un aprendizaje que crea una capacidad y puede ser utilizado diariamente de la vida. Aprender a tener hábitos de higiene da seguridad en los niños y niñas de enfermarse menos y estar saludable, adaptándose a sus preferencias personales, es la primera medida preventiva que debe practicar cualquier persona y que mejor empezando en la etapa inicial: ropa limpia, baño diario, lavarse las manos cada vez que vaya a comer y después de evacuar, enseñarle al niño como debe cambiar su ropa él solo, como debe lavar el plato, cuchara, vaso, taza, tenedor, su ropa, sus juguetes, limpiar la mesa, ordenar su cuarto, por ejemplo, enseñar donde ordenar sus juguetes, su habitación esté limpia, que sus juguetes estén limpios, el baño donde se asea esté limpio, o el lugar donde se limpia a un niño o niña, sí vas abrazar a un niño debes estar limpio de las manos y sí vas a dar de comer lávate las manos y los alimentos que preparas.

\section{Conclusiones}

Este estudio dio cuenta un currículo o propuesta para niños y niñas que acompañan a sus padres en albergues. Atender a los niños y niñas que se encuentran en estado de vulnerabilidad, es una prioridad, los docentes debemos contemplar el rol que deben tener para estas circunstancias. Los niños están vulnerables cuando llegan a un albergue, ya que no cuentan con servicios de guardería integral, en la cual no tienen una educación por la vía formal o no formal completa, que sea garante de sus derechos de los niños, con esta propuesta los encargados de los albergues tendrán una orientación de que hacer en los casos de cuidado de los niños y niñas de 0 a 6 años de edad.

El cuidado de los niños y niñas es vital para un futuro próspero para el infante, la cual conlleva a no enfermarse de cáncer, evitar violencia, y prevención de otras enfermedades que hace que mueran a temprana edad. 
Siempre se debe llevar a cabo un cuidado responsable de los niños y niñas, ya que son vulnerables, ya que no se cuidan solos, no tienen la capacidad de razonamiento del autocuidado, por lo tanto, necesitan de los adultos que se comprometan y sean responsables de su cuidado que por derecho debe ser una garantía.

Como investigadora, asesora y académica en la materia, es primordial contemplar el currículo para un desarrollo integral de los niños y niñas, como un compromiso para dar vida y alargar vidas, además aprendizajes básicos para el aprendizaje permanente. Cabe destacar que un currículo es vital para el desarrollo integral, es indispensable considerar la alimentación, ejercicios físicos, motores, afectivos y cognitivos continuos, como una crianza integral. Dicha propuesta se puede implementar por la vía formal y no formal, sin embargo, se necesita más ingresos a los albergues para cumplir las necesidades para llenar este vació de situaciones importantes para la vida de los niños y niñas.

La alimentación es vital para el desarrollo motor y físico del infante, por la cual, se debe dar los alimentos del plato del bien comer; es decir hacer platillos con la combinación de alimentos, vegetales, cereales y carne, del diario con cantidades acordes a lo que necesitan los niños pequeños, junto la jarra del buen beber, así como la leche materna, para dar vida entera. La cual es vital para todos los niños y niñas.

Es imprescindible el juego para el desarrollo psicomotor para que el infante desarrolle su cerebro, nervios, médula y músculos, sin ello puede ocasionarle graves problemas. Las actividades de la lectura, es primordial leer al infante de cero a seis años, para un desarrollo psicomotor, cognitivo, afectivo, psicológico y emocional en los niños y niñas. Las actividades de la lectoescritura, es importante no dejar de lado la enseñanza del español a partir del interés, madurez, y propiciar el gusto por la lectoescritura en los niños y niñas. 
Los cuidados de higiene se previenen enfermedades, y propicia un desarrollo integral. Por consiguiente, siempre tener la mirada hacia los niños para cumplir sus derechos por el bien de la humanidad y buen futuro de las nuevas generaciones. Para que se cumplan los derechos de los niños, necesitan ayuda de todos, con ayuda alimenticia, educativa, protección, referencia y atención temprana. Por lo tanto, la Universidad Pedagógica del Estado de Chihuahua, está comprometida con la niñez, educando las nuevas generaciones de docentes comprometidos, creando nuevas propuestas para un buen presente y futuro de la infancia.

\section{Referencias}

Alonso, D. (2018). Desarrollo de las habilidades motrices de las personas con discapacidad intelectual a través del proceso cognitivo. Artseduca, (19), 224-245, e-ISSN: 2254-0709. Recuperado de: http://dx.doi.org/10.6035/Artseduca.2018.19.10

Asociación Mundial de Educadores Infantiles (2006a,b). La educación de la primera infancia: Reto del siglo XXI. 2ad Edición, ISBN: 9789682476037. México: Editorial Trillas, S.A. de C.V.

Banco Mundial (2018). Según el Banco Mundial, la pobreza extrema a nivel mundial continúa disminuyendo, aunque a un ritmo más lento. Comunicado de Prensa N. ${ }^{\circ}$ 2019/030/DEC-GPV. Washington, Estados Unidos: Grupo Banco Mundial.

Brahm, P., \& Valdés, V. (2017a,b). Beneficios de la lactancia materna y riesgos de no amamantar. Revista chilena de pediatría, 88(1), 07-14, e-ISSN: 0370-4106. Recuperado de: https://dx.doi.org/10.4067/S037041062017000100001

Cabalé, E., \& Rodríguez, G. (2017). Educación no Formal: potencialidades y valor social. Revista Cubana de Educación Superior, 36(1), 69-83, eISSN: 0257-4314. Recuperado de: 
http://scielo.sld.cu/scielo.php?script=sci arttext\&pid=S0257$\underline{43142017000100007}$

Capurro, D., \& Rada, G. (2007). El proceso diagnóstico. Revista médica de Chile, 135(4), 534-538, e-ISSN: 0034-9887. Recuperado de:

https://dx.doi.org/10.4067/S0034-98872007000400018

Chacón-Ortiz, M. (2015). El proceso de evaluación en educación no formal: Un camino para su construcción. Revista Electrónica Educare, 19(2), 21-35, e-ISSN: 1409-4258. Recuperado de:

https://doi.org/10.15359/ree.19-2.2

Eming, M., \& Fujimoto, G. (2004). Desarrollo infantil temprano: lecciones de los programas no formales. Acción pedagógica, 13(2), 186-198, eISSN: 1315-401X. Recuperado de:

https://dialnet.unirioja.es/servlet/articulo?codigo $=2970476$

Faria, E., Oliveira, M., \& Ferreira, M. (2015). Hygiene care - bath: meanings and perspectives of nurses. Revista de Enfermagem Referência, IV(5), 37-44, e-ISSN: 2182.2883. Recuperado de:

https://dx.doi.org/10.12707/RIV14066

Gil, J. (2019). La lectoescritura como sistema neurocognitivo. Educación y Educadores, 22(3), 422-447, e-ISSN: 2027-5358. Recuperado de: https://doi.org/10.5294/edu.2019.22.3.5

Gutiérrez, R. (2018). Habilidades favorecedoras del aprendizaje de la lectura en alumnos de 5 y $\mathbf{6}$ años. Revista signos, 51(96), 45-60, eISSN: 0718-0934. Recuperado de: https://dx.doi.org/10.4067/S0718$\underline{09342018000100045}$

Iriondo, W., \& Gallego, D. (2013). El currículo y la educación a distancia. Ried, 16(1), 109-132, e-ISSN: 1138-2783. Recuperado de: https://doi.org/10.5944/ried.16.1.2062

Ley General de los Derechos de Niñas, Niños y Adolescentes (2018). Ley General de Prestación de Servicios para la Atención, Cuidado y 
Desarrollo Integral Infantil. Cuarta edición, ISBN: 978-607-729-472-6.

México: Comisión Nacional de los Derechos Humanos.

Maslow, A. (1943). A theory of human motivation. Psychological Review, 50(4), 370-396, e-ISSN: 1939-1471. Recovered from:

https://doi.org/10.1037/h0054346

Mendoza, M. (2017). Desarrollo de la motricidad fina y gruesa en etapa infantil. Sinergias educativas, 2(2), 1-7, e-ISSN: 2661-6661. Recuperado de: https://doi.org/10.31876/s.e.v2i2.25

Sarmiento, C. (2018). Fortalecimiento de los procesos de lectoescritura a través de la implementación de estrategias pedagógicas apoyadas en las TIC. Cultura, Educación y Sociedad, 9(3), 945-950, e-ISSN: 2389-7724. Recuperado de:

https://doi.org/10.17981/cultedusoc.9.3.2018.112

Soto, V., Gómez, O., Parrado, Y., Hernández-Rodríguez, P., \& Gomez, A. (2017). Caracterización de hábitos de higiene y ambientes en lugares de atención integral a población infantil. Revista da Escola de Enfermagem da USP, 51, 1-7, e-ISSN: ISSN 1980-220X. Recuperado de: http://dx.doi.org/10.1590/S1980-220X2016042103264 UNICEF (2018a,b). Informe Anual 2018. México, D.F.: Fondo de las Naciones Unidas para la Infancia. Recuperado de: https://www.unicef.org/mexico/media/1781/file/Informe\%20anual\%202 018.pdf 


\section{Mariela González López \\ e-mail: mgonzalez.ijd@upnech.edu.mx}

Nacida en Jalisco, México, el 2 de enero del año 1979.

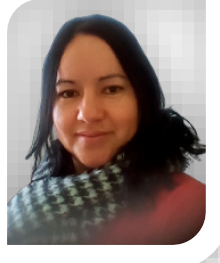

Pasión por la Investigación; Doctoranda en Educación Artes y Humanidades de la Universidad Autónoma de Chihuahua $(\mathrm{UACH})$, México; Maestra en la maestría en problemas de aprendizaje del Instituto José David (UPNECH); y en docente universitaria en el Instituto de Superación Integral Tultepec (ISITT); entre mis publicaciones recientes están: dos libros de texto de educación primaria (Matemáticas y de lectoescritura) con el título de Evaluación diagnóstica de habilidades de pensamiento e informativas para niños de primer grado de educación primaria (2019); la Alfabetización Informacional: enseñanza y desarrollo de su competencia en la educación básica" (2019); La enseñanza de habilidades de pensamiento y de la comprensión lectora en estudiantes de bajo rendimiento escolar (2019); y Las habilidades de pensamiento e informacionales en educación básica (2020), en el II Congreso Internacional de Jóvenes Investigadores; mis temas de interés son en educación básica, habilidades de pensamiento crítico, socioemocionales e informacionales; He participado en congresos nacionales e internacionales como ponente en temas como habilidades informacionales, educación básica, educación infantil, habilidades de pensamiento y pensamiento crítico; soy Certificada en Blackbelt, ISO 9000 e ISO 14000; con Diplomado en violencia sexual infantil y tutoría; además cuento con experiencia como maestra de educación primaria; Revisora de artículos de cinco revistas indizadas.

El contenido de este manuscrito se difunde bajo una Licencia de Creative Commons ReconocimientoNoComercial-Compartirlgual 4.0 Internacional 